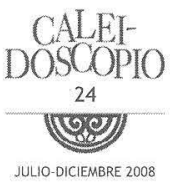

JULIO-DICIEMBRE 2008

\title{
La formación de los universitarios y la necesidad de considerar la formación en competencias
}

PATRICIA MEZA

Benemérita Universidad Autónoma de Puebla

En las actividades de Vinculación Universidad-Empresa se hace mención de la diferencia en el lenguaje, la diferencia en tiempos y otras importantes diferencias que separan el mundo de la universidad del de la empresa. Se ha escrito mucho sobre cómo conocer las necesidades de la empresa, para que la universidad contribuya a solucionar sus problemas y este acercamiento abra las puertas a los egresados universitarios.

Sabemos del elevado porcentaje de empresas micro y pequeñas en nuestro país en donde a menudo el egresado universitario está sobrecalificado para un puesto mecánico y repetitivo. Los empresarios se quejan de que los egresados llegan sin experiencia y que tienen que invertir en su capacitación. El egresado por su parte no tiene claro cómo es que podrá aplicar sus conocimientos, llega a un mercado laboral con gran demanda de conocimientos en ventas, con sueldos muy bajos y en donde siente que no aplica los conocimientos adquiridos y que además en pocos años serán obsoletos.

El empresario ve en el egresado "un ente económico" del que es pera sea polivalente, que sepa trabajar bajo presión, en equipo, por resultados y con actitud de servicio entre otras habilidades y actitudes. Ante estas consideraciones y muchas más para las cuales no hay espacio por el momento para enumerarlas, creemos que la universidad puede preparar mejor a sus egresados con una formación integral que 
incluya a las competencias; lo cual a su vez constituye una forma de hacer frente a retos que son fundamentales.

Un reto importante para la universidad es el de contribuir a la formación de seres humanos con valores, que fortalezcan su identidad, trasciendan, y sean capaces de incidir en un cambio permanente, con la mira en mejorar las perspectivas futuras.

También se debe preparar profesionistas en los saberes científicos, tecnológicos y humanísticos; con capacidades y experiencias mediante la formación alternada de prácticas profesionales y estadías que permitan la aplicación del saber en diferentes contextos, que generen cambios con el ejercicio de la profesión y conocimiento mediante la investigación.

La universidad debe proveer a sus alumnos de habilidades, capacidades y aptitudes. Habilidades que, agregadas a los conocimientos adquiridos y dentro del ejercicio profesional, le permitan al egresado hacer frente al desempleo existente como innovador y emprendedor que genere riqueza. Habilidades como el comunicarse o interactuar de manera efectiva, como la solución de problemas, el pensamiento crítico, el trabajo en equipo y otras tantas que intervienen en una perspectiva humanista en el desarrollo personal y profesional.

Diferentes manifestaciones y resultados señalan que los egresados universitarios no han desarrollado sus habilidades de aprender a aprender, pensar y crear; y que no están conscientes de las habilidades adquiridas, ni están capacitados para aplicar los conocimientos aprendidos. También se percibe que, de manera general, al alumno se le ha preparado para ser empleado y no innovador, emprendedor, creativo, reflexivo o crítico; y si a esto se suma la provisionalidad de los conocimientos, la voracidad de este mundo inequitativo, la pérdida de valores, la visión de inmediatez y utilitarismo así como el desempleo, todo esto conforma un difícil panorama al que se enfrenta el egresado universitario.

La adquisición de competencias por parte del alumno, implica adquirir los procedimientos y capacidades que le permitirán actualizarse y aprender de forma autónoma a lo largo de toda su vida, con habilidades -como elemento de las competencias- que en lugar de volverse obsoletas se desarrollen y aumenten y constituyan la base para aprender a aprender, pensar y crear, además de apoyar la competitividad y la 
empleabilidad. Es decir, que el capital de competencias adquirido le permita al egresado universitario mantener su nivel de desempeño en su vida personal, profesional y social. Por ello, y como un forma de contribuir al conocimiento de la realidad de nuestro entorno y al mismo tiempo de colaborar con un referente en el proceso del diseño del currículo por competencias, llevamos a cabo una investigación sobre los conocimientos, las habilidades y las actitudes que requiere el sector empresarial mexicano de los egresados universitarios de las diez profesiones más demandadas. ${ }^{1}$

Las diez profesiones mas demandadas son:

- Administración

- Contaduría

- Mercadotecnia

- Psicología

- Ingeniería Industrial

- Ingeniería Mecánica

- Ingeniería Química

- Ingeniería Civil

- Computación

- Informática

Síntesis de los resultados obtenidos sobre las variables generales

En lo que toca a la edad requerida, se observa una tendencia a solicitar gente más joven en Psicología, Administración e Informática, de 26 a 30 años. Por el contrario en las ingenierías (Industrial, Civil, Mecánica y Química) la edad solicitada varía de 31 a 35 años.

Este artículo presenta algunos apartados y síntesis de los resultados de la investigación de Patricia Meza, Las Competencias que requiere el Sector Empresarial Mexicano de los Egresados Universitarios, Instituto de Ciencias Sociales y Humanidades, Benemérita Universidad Autónoma de Puebla, México, 2008. 
De los resultados sobre el sexo preferido en los puestos vacantes se observa que si bien la suma de los conceptos "indiferente" y "no especificado" son elevados en todas las profesiones, se aprecia una preferencia por el sexo masculino en las Ingenierías y por el femenino en Psicología y Administración. También para Ingeniería Mecánica e Ingeniería Química se piden 5 o más años de experiencia. Se muestra lo contrario, menor exigencia en años de experiencia (uno a dos años) en: Psicología, Computación, Administración, Contaduría e Informática. En todas las profesiones predomina la demanda por el nivel de licenciatura y varía del $84 \%$ al $96 \%$ del total de sus ofertas de trabajo. Los puestos vacantes que se ofertan a pasantes, se encuentran sobre todo en: Informática, Administración y Contaduría en donde representan del $6 \%$ al 10\%. Son las cuatro ingenierías las únicas en donde la demanda por niveles de maestría manifiesta porcentajes significativos, bien que estos sean bajos: del 6.3\% en Ingeniería Mecánica al 9.9\% en Ingeniería Civil. En cuanto al sueldo ofrecido este es superior en las cuatro Ingenierías, Mercadotecnia y Computación y más bajos en Psicología, Contaduría y Administración.

Los requerimientos de Idiomas- expresados en porcentajes del total de las ofertas de trabajo para cada profesión - son más elevados en: Ingeniería Química 63\%, Ingeniería Industrial 60\% e Ingeniería Mecánica 58\%. Se considera que las exigencias en Idiomas son bajas para Computación e Informática - 41\% y 32\% respectivamente - si se tiene en cuenta las características del desenvolvimiento profesional de estas dos carreras.

A continuación se presentan los conocimientos que requiere el sector empresarial mexicano de los egresados en Administración, como un ejemplo de los resultados que contiene el documento original para cada una de las diez profesiones de estudio.

\section{Conocimientos requeridos para Administración}

Del conjunto de conocimientos que el sector empresarial de las cuatro entidades federativas de estudio demanda de los egresados de Administración, los más importantes son:

- Recursos Humanos

- Contabilidad 
- Ventas

- Administración

Esto se puede apreciar en la gráfica 1, la cual también nos muestra como los Recursos Humanos ocupan el primer lugar con el 11\%. Sin embargo, si a estos se agregan: Reclutamiento y Selección de Personal (con el 4\% y 3\% respectivamente), Capacitación (3\%), Exámenes Psicométricos ( $1 \%$ ) y Administración de Personal (4\%); se llega a un 26 $\%$. Es decir, una cuarta parte del total de los conocimientos requeridos de la carrera de Administración están relacionados con los recursos Humanos.

En lo que toca a los conocimientos relacionados con Contabilidad, estos representan un 19\% del total, integrados por: Contabilidad $8 \%$, Aportaciones a la Seguridad Social (IMSS e INFONAVIT) (3\%), Nómina (4\%), Facturación (1\%), Cobranza (1\%), Impuestos (1\%) y Seguros (1\%). Por otra parte, como se mencionó anteriormente, los conocimientos relativos a las Ventas son importantes al sumar 15\% del total de los conocimientos requeridos para los profesionistas en Administración .Dicha suma se compone de: la venta del producto como tal (8\%) y del Análisis de Ventas (3\%); este último integrado a su vez por: planes, proyecciones y estrategias de ventas. Finalmente agregaríamos a esta área de ventas, el $4 \%$ que corresponde a los conocimientos sobre Clientes.

En lo que refiere a Administración, los conocimientos requeridos y relacionados con esta área son: Administración (8\%), Administración Financiera (2\%) y Administración de Inventarios (2\%). Esta suma asciende a $11 \%$. Casi con el mismo nivel de importancia se encuentran las áreas de Derecho (4\%), Mercadotecnia (4\%), Compras (4\%) y Logística (3\%). Finalmente para el conjunto de las cuatro entidades de estudio, se considera que para los empleadores los conocimientos solicitados sobre Calidad (1\%) tienen poca importancia. 


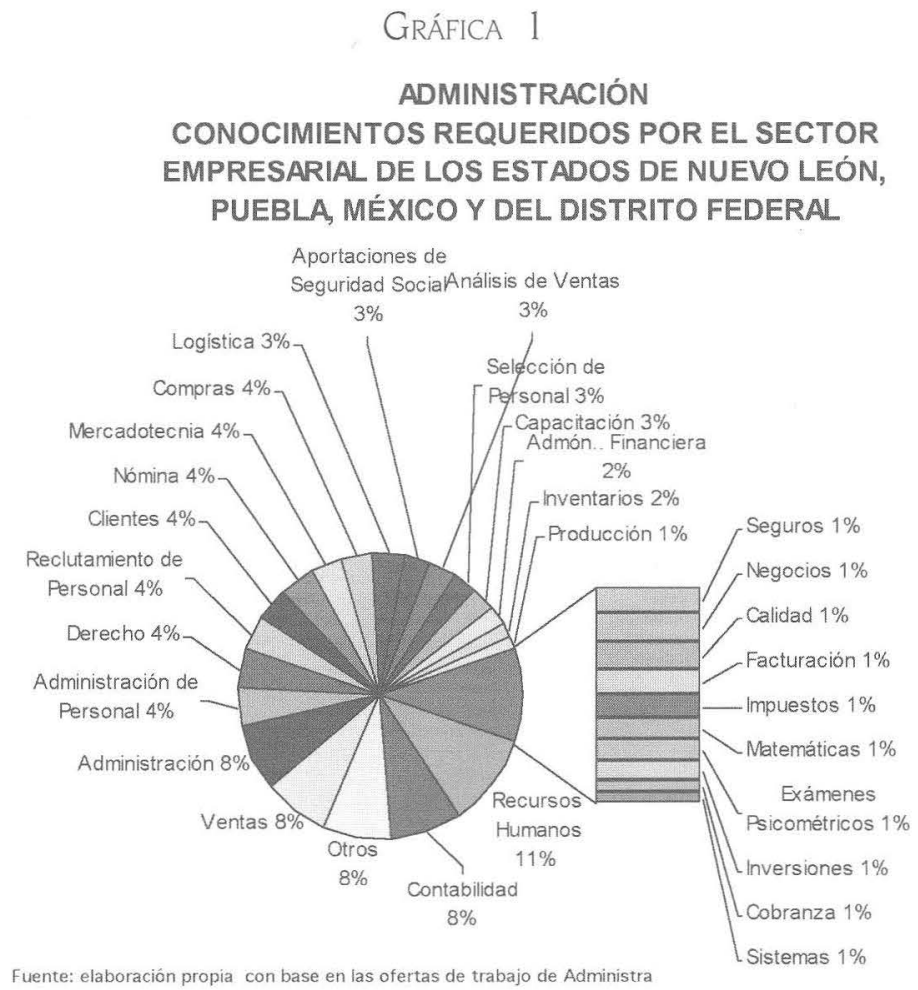

En la grafica 2 "Herramientas requeridas para la carrera de Administración de Empresas", se observa que más de las dos terceras partes $(86 \%)$ del total requerido por los empleadores, corresponden al Sistema de Paquetería de Office, dentro del cual se encuentran: Word (17\%), Excel (21\%), Power Point (16\%), Access (16\%) y Outlook (16\%). Le sigue en importancia el software de Gestión Empresarial Integrada (ERP) con el 8\%; a este porcentaje participa el software administrativo de empresas como son: ASP( $2 \%)$, ASPEL (2\%), People Soft ( $1 \%)$, Baan (1\%), JD Edwards (1\%) y Oracle (1)\%. Finalmente los empleadores solicitan Manejo de PC y del Sistema Operativo Windows con $2 \%$ y $1 \%$, respectivamente. 


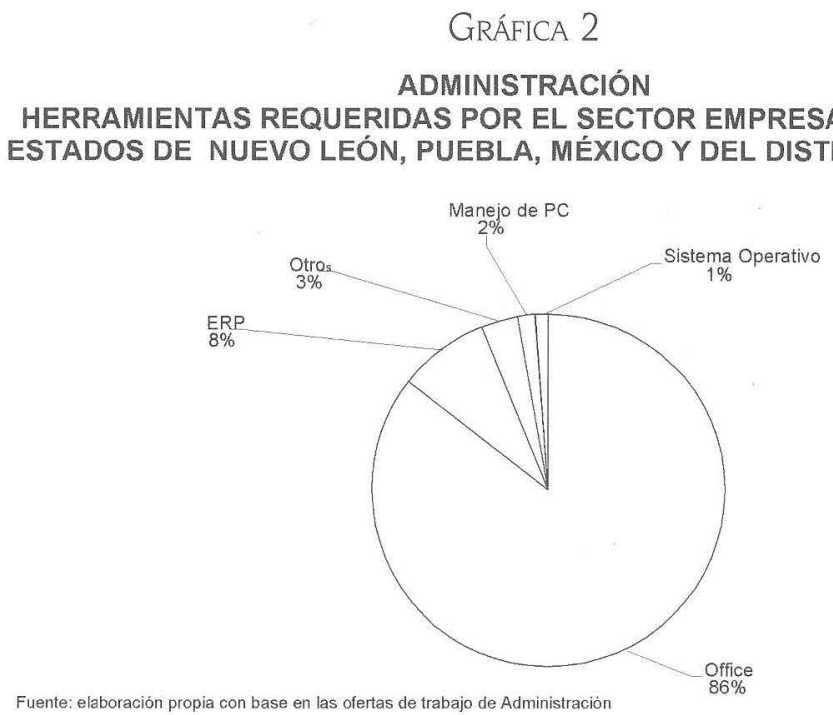

El siguiente esquema muestra los conocimientos con mayor demanda por parte del sector empresarial de las cuatro entidades de estudio para cada una de las 10 profesiones. Estos conocimientos se "despliegan" en el documento original como se acaba de mostrar en el ejemplo de Administración

CONOCIMIENTOS MAS DEMANDADOS POR PROFESION

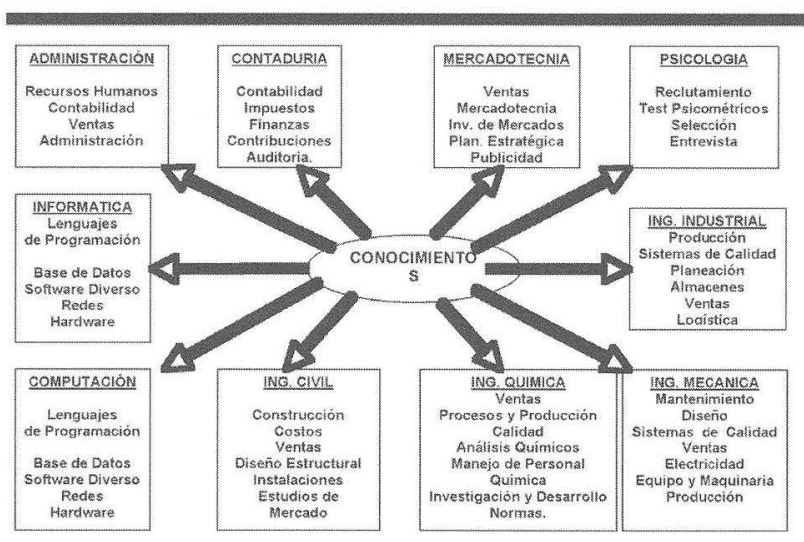

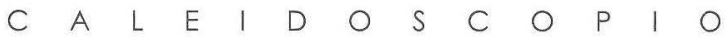


A continuación se agregan algunas precisiones que se toman de la observación de las gráficas de cada profesión que contiene el documento original.

\section{Administración}

- La cuarta parte (26\%) del total de los conocimientos requeridos, están relacionados con los Recursos Humanos y el 15\% con el área de Ventas.

\section{Contaduría}

- No se le da la importancia suficiente al Control Interno como un área esencial del buen funcionamiento de la empresa.

\section{Mercadotecnia}

- El $41 \%$ del total de los conocimientos requeridos se integra por Promoción, Investigación de Mercados y Estadística.

\section{Psicología}

- El 82\% del total de conocimientos requeridos corresponden a áreas directamente relacionadas con la Psicología Organizacional.

\section{Ingeniería Industrial}

- El 33\% del total de conocimientos solicitados (Producción, Sistemas de Calidad y Planeación) es congruente con el objetivo de obtener un producto de calidad en el tiempo indicado.

\section{Ingeniería Mecánica}

- El 29\% del total de conocimientos (Producción, Calidad, Mantenimiento y Equipo y Maquinaria), son necesarios en la elaboración de productos manufacturados que deben seguir parámetros de calidad que se logran con un adecuado mantenimiento de las máquinas y buenos programas de producción.

Ingeniería Química

- De las 28 áreas de conocimientos demandados a los egresados de 
esta carrera, la primera y más importante es el área de Ventas y la quinta el Manejo de Personal.

\section{Ingeniería Civil}

- Una cuarta parte del total de los conocimientos requeridos de esta carrera está relacionada a las áreas Administrativo - Contables, necesarias a la Construcción y se solicitan conocimientos sobre Ventas y Atención a Clientes en una proporción importante como es el $7 \%$.

\section{Computación e Informática}

- Los empleadores demandan los mismos conocimientos tanto a Computación como a Informática.

- Sin embargo, estas dos profesiones son diferentes curricularmente ya que el perfil profesional de Informática está diseñado para administrar y mantener servicios y sistemas de información integrados y eficientes que faciliten la toma de decisiones y agilicen las operaciones de una organización.

- Por otra parte, el perfil profesional de Computación está dirigido al diseño de modelos de construcción para la industria del Software, a la aplicación de los modelos formales de Cómputo, técnicas de diseño y análisis de algoritmos.

La importancia de las áreas de Ventas, Recursos Humanos y Manejo de Personal

- Solo en cuatro de las 10 profesiones de estudio, (Contaduría, Psicología, Computación e Informática) las ventas no son significativas; sucede lo contrario en las seis restantes. Así, de un promedio de 27 áreas que aparecen en las gráficas de Conocimientos de las diferentes profesiones; el área de Ventas, en Mercadotecnia e Ingeniería Química ocupa el primer lugar; en Ingeniería Civil el segundo, en Administración el tercero, en Ingeniería Industrial el quinto y en Ingeniería Mecánica el sexto.

- La suma promedio de las áreas de Recursos Humanos y de Manejo de Personal asciende a un importante 6\% del total de los conocimientos demandados en las Ingenierías de estudio (Industrial, Mecánica, Química y Civil). 
- Lo anterior se explica por la competencia internacional a la que se enfrentan las empresas, las cuales requieren que su personal maneje las Tendencias Comerciales; que unidas a la nueva Cultura Laboral nacional -enfocada al desarrollo humano - hace que los empresarios estén a la búsqueda de profesionistas que garanticen el buen Manejo de Personal y que conozcan del Trabajo en Equipo y Enfoque al Cliente

\section{Herramientas}

Las participaciones más importantes - 21\% al $86 \%$ - del total de las Herramientas requerida en cada profesión de estudio; corresponden a Office con sus diferentes módulos (Word, Excel Power Point, Access y Outlook). Sobresale también el Software de Gestión Empresarial Integrada, ERP, que en el caso de la carrera de Administración interviene como Software Administrativo de empresas como son: ASP, ASPEL, People Soft, Baan y ID Edwards. En Contaduría, el Software ERP representa el 50\%, porcentaje en el que participan además empresas como: SAP, Hyperion y Mfg.Pro. En la carrera de Psicología, pero sobre todo en las Ingenierías: Industrial, Química y Mecánica se requieren conocimientos en ERP, principalmente SAP. En las diferentes carreras se llega a demandar Software ERP como tal, sin especificar empresa alguna.

En Mercadotecnia, la Herramienta más demandada es el Software de Diseño, de empresas como Adobe (en donde destaca Photoshop) y ACNielsen, líder en investigación, información y análisis de mercado. Los Programas de Diseño como Autocad, Autocad 2 y 3D, Studio Max , CAD-CAM y Modelado en 3D aparecen en Ingeniería Industrial, Mecánica y Civil. En Ingeniería Industrial se solicitan principalmente Sistemas de Gestión de Bases de Datos y Programas de Análisis Estadístico como Minitab, y MRP.

En Ingeniería Química, se requiere de Control de Calidad; en este caso se trata de un Sistema de Análisis de Riesgos y Puntos Críticos de Control (HACCP). En Ingeniería Mecánica, las principales Herramientas requeridas en Software se refieren a conocimientos en: CNC - Control Numérico Computarizado - Microstation y CAE (Ayuda Computacional 
a la Ingeniería). Para Ingeniería Civil se solicita Software para Administración y Control de Proyectos y Obras (Staad, StaadiII y Staad Pro) y Software para Arquitectura e Ingeniería (Microstatio, Neodata, Opus, Primavera, Icaro y Suretrak).

De la revisión de las gráficas de Herramientas se observa una marcada tendencia a solicitar conocimientos sobre la paquetería de Office. Al respecto se debe mencionar que Excel fue concebido como una hoja de cálculo con ciertas funciones de base de datos, lo que no le convierte en un Sistema Gestor de Bases de Datos (SGBD) y en este sentido, tiene desventajas en relación a Access. Sentimos que Access tiene una demanda muy baja en el total de requerimientos, y que se está desperdiciando su potencial en la solución de problemas administrativos y que en su lugar se utiliza Excel creando soluciónes inadecuadas.

Las Habilidades más Solicitadas de Acuerdo a su Incidencia dentro del Total de las Ofertas de Trabajo. ${ }^{2}$

De las 21 Habilidades más requeridas, solo se consideraron 14, las siete restantes además de tener una muy baja incidencia no se encuentran presentes en todas las profesiones. Las 14 Habilidades más demandas quedaron agrupadas en tres bloques:
A.- Incidencia Alta
1.-Trabajo bajo Presión
2.- Trabajo en Equipo
3.-Liderazgo
4.-Facilidad de Palabra
5.-Negociación

Sobre el tema de las habilidades de empleabilidad un importante artículo es el de Kathleen Cotton, Developing Employability Skills, School Improvement Research Series (SRS), 1993. 
B.- Incidencia Media

6.-Relaciones interpersonales

7.- Capacidad de Análisis

8.-Trabajo por Resultados

9.-Trabajo por Objetivos

10.- Organización (no aparece en Ingeniería Química)

C.- Incidencia Baja

11.- Iniciativa

12.- Toma de Decisiones

13.- Comunicación

14.- Solución de Problemas

Las Actitudes y Valores más solicitados de acuerdo a su incidencia dentro del total de las Ofertas de Trabajo

De 31 Actitudes y Valores más solicitados resultaron ser 8 los de mayor incidencia y quedaron agruparon en 3 bloques:

A. - Incidencia Alta

1.-Excelente Presentación

2.-Pro-Actividad

3.-Actitud de Servicio

B.- Incidencia Media

4.- Responsabilidad

C.- Incidencia Baja

5.- Compromiso

6.-Dinamismo

7.- Honestidad

8. - Orden.

Consideraciones finales

En un inicio dijimos que la Universidad debe contribuir a la formación de seres humanos con valores, de preparar profesionistas en saberes científicos, tecnológicos y humanísticos. Mencionamos la importancia de la experiencia y la formación en alternancia como una opción para adquirir dicha experiencia. Se anotó que la universidad debe proveer a sus alumnos de habilidades, capacidades y aptitudes que le permitan 
al egresado hacer frente al desempleo existente como innovador y emprendedor que genere riqueza.

Creemos que la Universidad debe integrar el enfoque de competencias al diseño curricular de una manera apropiada, partiendo de su Misión, tratando de cumplir con su compromiso en el desarrollo del entorno y con sus metas y objetivos en la calidad académica; concediéndole igual importancia a la formación humanista y a la conciencia social y crítica del egresado. ${ }^{3}$

Insistimos en que si bien se debe tener presente la función de vinculación del sistema educativo con el sistema económico, y la necesidad de conocer las competencias necesarias para el aseguramiento de la productividad y la competitividad; no se debe limitar la adquisición y transmisión de competencias a la eficiencia del sistema económico. Porque los individuos no son solamente actores económicos sino que asumen múltiples papeles en la sociedad, en la política, la cultura, la familia y demás interrelaciones personales. Esas capacidades como ciudadanos les servirán tanto a su calidad de vida personal como a construir cualidades colectivas y contribuir a la integración y cohesión social como la solidaridad, la comunicación intercultural y el respeto de los derechos humanos. Para nosotros una política de educación y promoción de competencias debe tener por objetivo la búsqueda de la igualdad de oportunidades y la contribución a la realización efectiva de los derechos humanos.

Estamos convencidos de que para trabajar en la formación en competencias, además de conocer las experiencias, modelos y tipologías de otros países, se debe conocer la realidad que nos rodea como punto de partida en un proceso de transformación y mejora. Convencidos de lo anterior llevamos a cabo la investigación mencionada al inicio de este artículo, cuyos resultados esperamos constituyan un referente en el trabajo de definición, clasificación, desarrollo e implementación de las competencias en la Universidad.

(2)

Sobre los grandes cambios en organización y metodología que conlleva la enseñanza en "contacto" con las competencias ver : Margot Phanehf, Quelaues réflexions sur le role de l'enseignante dans une approche par compétences, http//www.infiressources.ca/fer/ depot documents, 2003. 
